\title{
Wie verschleiert China den Protektionismus?
}

\author{
Piotr Lukaszuk
}

\section{Relevanz}

Wenn Freihandel und Wettbewerb zusammenspielen, können sich die Länder am ehesten auf das spezialisieren, was sie am besten können. Alle gewinnen. Doch die protektionistische Verlockung ist groß, die eigene Wirtschaft auf Kosten anderer zu schützen. Dem soll die WTO einen Riegel vorschieben, indem sie Importzölle und andere Handelsbeschränkungen weltweit abbaut. Doch der Einfallsreichtum der Protektionisten kennt keine Grenzen. Sie weichen unter anderem auf Exportbeschränkungen aus. Kann es protektionistisch sein, den Export einzelner Branchen zu behindern und damit den Markt anderen Ländern zu überlassen? Der Schachzug besteht darin, mit Export- und Absatzbeschränkungen den Preis in dieser Branche $\mathrm{zu}$ drücken und damit die heimischen Vorleistungen für die eigentlich strategisch wichtigen Branchen zu verbilligen! Auch damit kann man den Schlüsselbranchen auf dem Weltmarkt einen unfairen Wettbewerbsvorteil verschaffen. Diese gelenkte Industriepolitik ist protektionistisch und weltweit im Vormarsch, in China und in anderen Ländern.

\section{Quelle}

Jason Garred (2018), The Persistence of Trade Policy in China after WTO Accession, Journal of International Economics 114, S. 130-142.

P. Lukaszuk $(\bowtie)$

Universität St.Gallen, St.Gallen, Schweiz

E-Mail: piotr.lukaszuk@student.unisg.ch 
In den letzten Jahrzehnten konnte die Welthandelspolitik Einfuhrzölle systematisch und weltweit abbauen. Gemäß der Weltbank sank der durchschnittliche Einfuhrzoll von rund $8.6 \% 1994$ auf $2.6 \%$ 2017. Hinzu kommen über 300 Freihandelsverträge, welche mittlerweile mehr als die Hälfte des Welthandels umfassen. Gleichzeitig weisen immer mehr Studien darauf hin, dass der Protektionismus wieder zunimmt und neue Handelsschranken entstehen - und dies schon lange vor Donald Trump. Wie passt das zusammen?

Nachdem die traditionellen Instrumente wie Einfuhrzölle zunehmend verpönt sind, haben sich die protektionistischen Tendenzen systematisch hin zu nicht-tarifären Barrieren wie Steuern, Subventionen oder Exportbeschränkungen verlagert. Letztere sind oft schwieriger festzumachen und gelten daher als „verschleierter Protektionismus". Gemäß dem Global Trade Alert, der weltweit größten Datenbank zu Protektionismus, haben sich die nicht-tarifären Handelsbarrieren zwischen 2009 und 2017 fast verdoppelt.

Der durchschnittliche Einfuhrzoll ist von rund 8.6 Prozent 1994 auf 2.6 Prozent 2017 gefallen. Dagegen haben sich die nicht-tarifären Handelsbarrieren von 2009 bis 2017 fast verdoppelt.

Die Studie von Jason Garred von der Universität Ottawa untersucht am Beispiel Chinas den Wandel von Einfuhrzöllen zu nicht-tarifären Barrieren und ihre protektionistischen Auswirkungen. Der Forscher hat nicht bloß die üblichen Handelszölle auf Importe und Exporte einbezogen, sondern weitere Beschränkungen wie Exportlizenzen und -verbote und Mehrwertsteuerrabatte für Exporte erfasst. Letztere spielen in Chinas Handelspolitik eine sehr wichtige Rolle. Während die meisten Länder ihre Exporte von der Mehrwertsteuer befreien, versteuert China grundsätzlich alle Exporte mit einem Steuersatz von $17 \%$. Seit 2004 wird aber die Ausfuhr mancher Produkte teilweise oder gänzlich von der Mehrwertsteuer befreit, um bestimmte Branchen zu fördern. Der kanadische Wissenschaftler kombiniert in seinem Aufsatz diese unterschiedlichen Exportbeschränkungen und berechnet damit ein Maß der effektiven Ausfuhrzölle für jedes Produkt.

Nach dem Beitritt zur Welthandelsorganisation (WTO) im Jahr 2001 passte China seine Schutzpolitik systematisch an, indem es Einfuhrzölle durch Ausfuhrhindernisse ersetzte. Die Ergebnisse wecken große Zweifel, dass die WTO-Mitgliedschaft tatsächlich Chinas Handelspolitik liberalisieren konnte. Abb. 1 zeigt, dass China nach 1997 nicht nur seine Einfuhrzölle stark senkte, sondern auch die Unterschiede der Zölle für verschiedene Produktkategorien halbierte. Dadurch werden heute die meisten Importe mit einem ähnlich hohen Zoll belastet. Bei den effektiven Ausfuhrzöllen zeigt sich jedoch gerade das Gegenteil: manche Produkte weisen nach 2006 starke Exportbeschränkungen auf, andere hingegen nicht. 


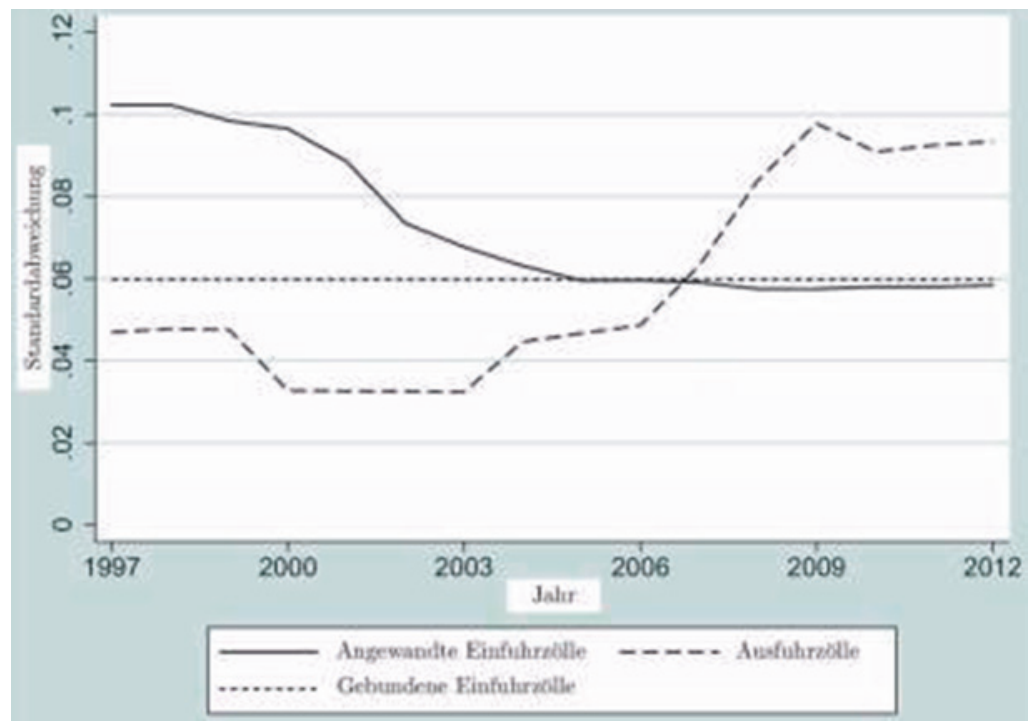

Abb. 1 Importzölle und Ausfuhrbeschränkungen in China (alle Produktkategorien ohne Agrarprodukte). (Quelle: Garred 2018)

China betreibt eine gelenkte Industriepolitik und entscheidet vorab auf höchster politischer Ebene, welche Branchen strategisch gefördert werden sollen. China schützt also nicht alle Branchen in gleicher Weise vor ausländischem Wettbewerb, sondern behandelt einzelne Produkte und Sektoren sehr unterschiedlich. Mit Importzöllen und anderen Barrieren kann es den Marktzugang für ausländische Güter behindern, während Exportbeschränkungen gezielt den Weltmarktzugang einzelner, lokal produzierter Güter erschweren. Um diese Strategie nachzuweisen, sind zwei Fragen zu beantworten: Erstens, inwieweit werden Einfuhrzölle durch Ausfuhrhürden ersetzt? Zweitens, welche Unterschiede gibt es bei Exportbeschränkungen zwischen verschiedenen Sektoren der chinesischen Wirtschaft?

Man kann eine Branche fördern, indem man sie entweder vor Importen schützt oder von Exportbeschränkungen ausnimmt. Mit dem WTO-Beitritt 2001 musste China Einfuhrzölle und nicht-tarifäre Handelsbeschränkungen abbauen. Jason Garred untersucht nun, ob Produkte, welche vorher mit hohen Einfuhrzöllen vor ausländischer Konkurrenz stark geschützt wurden, auch nach dem Beitritt eine weitere Bevorzugung erhielten, z. B. mit einer Ausnahme von einer allgemeinen 
Erhöhung von Ausfuhrzöllen. Seine Schätzungen zeigen, dass die effektiven Ausfuhrzölle zwischen 2002 und 2012 tatsächlich signifikant schwächer anstiegen, wenn in dieser Branche der Einfuhrzoll vor dem WTO-Betritt hoch war.

War der Einfuhrzoll eines Produkts vor dem WTO-Beitritt um 1 Prozentpunkt höher, fiel der Anstieg der Ausfuhrzölle um 0.5 Prozentpunkte geringer aus.

Eine strategisch wichtige Branche kann man auch fördern, indem man ihre Vorleistungen z. B. für die benötigten Rohmaterialien verbilligt. Ein Weg dazu ist, die Branchen in der vorgelagerten Wertschöpfungskette mit hohen Ausfuhrzöllen zu belegen. Die empirische Analyse zeigt, dass China neue Ausfuhrzölle vor allem auf Rohmaterialien und Halbfertigprodukte erhöhte. Die hohen Exportkosten beschränken den Auslandsabsatz der Branche und drücken den Preis. Die Produzenten von Rohmaterialien und Halbfertigprodukten müssen also ihre Erzeugnisse günstiger auf dem chinesischen Heimmarkt verkaufen. Vor allem bei Materialien, wo China signifikante Marktanteile besitzt und der Weltmarktpreis aufgrund des geringeren chinesischen Angebots steigt, entsteht dadurch eine Preislücke zwischen dem chinesischen und dem Weltmarktpreis. Damit erhält die nachgelagerte Schlüsselbranche in China gleich einen doppelten Wettbewerbsvorteil. Nicht nur kann sie im Inland billigere Vorleistungen beziehen. Gleichzeitig verteuern sich dieselben Vorleistungen für die ausländischen Konkurrenten.

Um diese Zusammenhänge empirisch zu belegen, vergleicht Jason Garred zunächst die Einfuhrzölle auf Fertigprodukte im Jahr 1999 mit den effektiven Ausfuhrzöllen auf Rohmaterialien nach dem WTO-Beitritt 2001, welche für die Herstellung genau jener Fertigprodukte notwendig sind. Seine Schätzungen zeigen, dass die Absenkung der Einführzölle Eins-zu-Eins mit einem Anstieg der effektiven Ausfuhrzölle einherging. Weiter zeigt er, wie der Einsatz von Ausfuhrzöllen die chinesischen Exporte beeinflusste. Steigt der effektive Ausfuhrzoll auf Rohmaterialien um 1 Prozentpunkt, gehen die Exporte dieser Branche um rund $5.1 \%$ zurück. Das löst einen Preisverfall im Inland aus, welcher den nachgelagerten Verarbeitungsstufen zugutekommt. Die Verbilligung der Vorleistungen steigert die Exporte der Fertigprodukte um bis zu $7 \%$.

Ein Anstieg der Ausfuhrzölle auf Rohmaterialien um 1 Prozentpunkt senkt die Exporte dieser Branche um 5.1 Prozent. Der ausgelöste Preisverfall verbilligt die Vorleistungen für die nachgelagerte Produktion und steigert die Exporte von Endprodukten um bis zu 7 Prozent. 
Die chinesische Regierung lässt sich weniger von Partikularinteressen beeinflussen, sondern betreibt offensichtlich eine zentral gelenkte Industriepolitik. China identifiziert strategisch wichtige Schlüsselindustrien, die sie mit verschiedenen Instrumenten gezielt subventioniert. China fördert diese Branchen, indem die Regierung ausländische Direktinvestitionen hemmt und gleichzeitig die Branche von Exportzöllen entlastet. Zusätzlich verbilligt sie die Vorleistungen der heimischen Produzenten, indem sie höhere Exportzölle auf den vorgelagerten Stufen erhebt. Indem diese Exportzölle tendenziell die Weltmarkpreise für die Rohmaterialien steigen lassen, benachteiligen sie zudem die ausländischen Konkurrenten. In anderen Branchen, die keine strategische Bevorzugung genießen, lässt China ausländische Direktinvestitionen zu und verzichtet auf den Einsatz von diskriminierenden Exportzöllen.

Diese Erkenntnisse werfen die Frage auf, ob die Politik, Einfuhrzölle durch Ausfuhrzölle zu ersetzen, nicht gegen WTO Regeln verstößt. Die Vereinigten Staaten riefen deshalb die WTO an, welche 2013 entschied, dass China Exportrabatte für bestimmte Produkte zurückziehen muss. Dieser Fall macht die Beschränkungen eines multilateralen Rahmens wie der WTO deutlich, welcher die Schutzpolitik ihrer Mitglieder einschränken soll. Auch andere WTO-Mitgliedstaaten wie Brasilien oder Indonesien setzen gezielte Ausfuhrzölle ein, um aktive Industriepolitik zu betreiben.

Die Gefahr solcher schutzpolitischen Maßnahmen, welche innerhalb der WTO-Gespräche nicht vollständig verboten werden können, liegt in der Ausbreitung. Wenn ein Land den Anfang macht, überlegen sich auch andere Länder, ähnliche Maßnahmen einzuführen, um gleiche Wettbewerbsbedingungen für ihre eigenen Firmen zu erhalten. Auch innerhalb der Europäischen Union werden die Stimmen für eine eigene Industriepolitik lauter, um mit den chinesischen und amerikanischen Unternehmen im Wettbewerb bestehen zu können. Am Ende verlieren alle. Alle müssen teure Subventionen finanzieren, aber kein Land erzielt einen Wettbewerbsvorteil, wenn die anderen nachziehen. Ein multilaterales Vorgehen im Rahmen der WTO wäre besser. 
Open Access Dieses Kapitel wird unter der Creative Commons Namensnennung 4.0 International Lizenz (http://creativecommons.org/licenses/by/4.0/deed.de) veröffentlicht, welche die Nutzung, Vervielfältigung, Bearbeitung, Verbreitung und Wiedergabe in jeglichem Medium und Format erlaubt, sofern Sie den/die ursprünglichen Autor(en) und die Quelle ordnungsgemäß nennen, einen Link zur Creative Commons Lizenz beifügen und angeben, ob Änderungen vorgenommen wurden.

Die in diesem Kapitel enthaltenen Bilder und sonstiges Drittmaterial unterliegen ebenfalls der genannten Creative Commons Lizenz, sofern sich aus der Abbildungslegende nichts anderes ergibt. Sofern das betreffende Material nicht unter der genannten Creative Commons Lizenz steht und die betreffende Handlung nicht nach gesetzlichen Vorschriften erlaubt ist, ist für die oben aufgeführten Weiterverwendungen des Materials die Einwilligung des jeweiligen Rechteinhabers einzuholen.

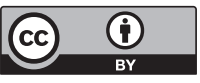

